\title{
RESOURCE REVIEW
}

\section{An Overview of the Pingo Audience Response System in Undergraduate Mathematics and Statistics Teaching}

\author{
Kristian Evans, Mathematics Department, Swansea University, Swansea, UK. Email:
}

K.Evans@Swansea.ac.uk

\section{Abstract}

Obtaining interaction and engagement in large group teaching can be challenging, in particular in STEM subjects where it is essential to be able to efficiently present symbols, equations and formulae. Pingo is a free, web-based system that provides an excellent way of introducing interaction in large group teaching, as there are no restrictions on the number of participants. It is particularly useful in Mathematics-based subjects as it is TeX compatible. Such interaction also provides feedback to students and lecturers and it is a straightforward, but creative way to enhance student engagement. The author has tried and tested Pingo weekly in Mathematics and Statistics modules. This article provides all the information and resources required to start using Pingo along with some reflections on its use in the Mathematics Department at Swansea University.

Keywords: Large group teaching, audience response system, Pingo, TeX, LaTeX, STEM.

\section{Introduction}

For the last two academic years I have trialled the Pingo audience response system with the aim of increasing participation, providing better, and more useful, feedback, teaching in a more inclusive way and for generally providing a better student experience. The main reason I wanted to do this was because I felt that there was a lack of interaction in lecture style teaching. Students often would be reluctant to ask questions or provide feedback during large group teaching activities. I believe that this is to the detriment of students' learning experience and I therefore decided to research techniques to overcome this. Initially, I was led by ideas and evidence in Bruff (2009) that interactive systems like Pingo help achieve this. Furthermore, I found the work by Bates, Howie and Murphy (2006) interesting, in particular the statement "The challenge is, therefore, to try to actively engage the students in the lecture, to develop it to be something more akin to a two-way conversation than a one-way transmission of information."

There are a number of audience response systems available to facilitate interaction in large group teaching environments, in particular Shon and Smith (2011) give an interesting account into the use of Poll Everywhere. Other systems include Zeetings, Socrative and Mentimeter. After researching many such systems, I decided to trial the use of Pingo due to a combination of the following reasons: it is TeX compatible, it is fee-free and there are no restrictions on the number of participants. TeX, or in its more advanced form including macros, LaTeX, is an open-source programme used by many academics in STEM-based subjects to typeset mathematical documents. In fact, many universities now require Mathematics undergraduates to be able to produce documents in LaTeX. Furthermore, the London Mathematical Society states in their Position Statement (London Mathematical Society, 2011), that "We need to reject ICT products not suited to writing, presenting and processing mathematical texts. LaTeX is a recognised solution, and students should be encouraged, and where possible taught, to use LaTeX to present mathematics in reports and projects." Pingo can also be used in a text-only way, however, one of its main advantages, in my opinion, is that it can be used to create advanced, mathematically-based questions due to its TeX compatibility.

Pingo was created and is run by the University of Paderborn in Germany. It is a web-based system and therefore only requires the lecturer to have access to an on-line computer and the audience to 
have access to an on-line mobile phone, tablet or computer. A further benefit of Pingo is that members of the audience do not need to sign up to use the system; only lecturers have to open an account in order to create questions. Questions may be asked in several ways in Pingo, varying from single and multiple-choice questions to open-ended questions requiring a text or TeX-coded answer. Similar to other audience response systems, the audience is given a set amount of time to answer a question, and once this time has elapsed the anonymous results appear on the screen for all to see. This provides instant feedback to both the student and the lecturer, and, where necessary, further information may be provided by the lecturer to explain any troublesome questions. Pingo also has a repeat feature which is useful to check that the audience now does understand a particular question. In my opinion, the anonymous nature of answering questions is important, as the audience is more likely to participate.

I have used Pingo in a third year module in complex analysis and a first year module in applied statistics. In both modules, I used the system during the final lecture of each week as an interactive method to test and refresh the topics taught that week. The majority of the questions I used were either multiple choice or single choice and mathematical or statistical in nature. However, I would also ask the following open-ended question every week: Are there any topics that you would like to go back over? If so, please state them below. For any comments listed, I would spend some time during the next lecture to revise these topics. Not only does this provide instant feedback to the lecturer, but it also facilitates prompt feedback - rather than having to wait for module questionnaire or survey results. I believe the overall learning and teaching experience has been enhanced by this intervention, reflecting the statement "The use of interactive lectures can promote active learning, give feedback to the teacher and the student, and increase satisfaction for both." in Steinert and Snell (1999).

The system has been well received by students, as evidenced by module questionnaire comments and comments in staff-student consultative committee meetings which are discussed in Section 3 below.

\section{Guide to Using Pingo}

This guide is written from an academic perspective with the intention of lecturers/teachers using Pingo to create an interactive learning environment for their students. Clearly, this could be adapted to other scenarios where interaction is desirable. More detailed information about the features of Pingo can be found on the developer's website or their YouTube channel.

- As mentioned in the Introduction, lecturers are the only users who have to sign up for a Pingo account, and this is achieved by visiting http://trypingo.com.

- Once signed up, the website that both staff and students use is http://pingo.upb.de. On this page, lecturers sign in to create questions and sessions whereas students input the access number (see later) for a particular lecture to participate in the session.

- Questions may be created on-the-spot, or a catalogue of questions may be created in advance. To do this, select Survey Design followed by New Question to get the following (Figure 1): 


\section{Create question}

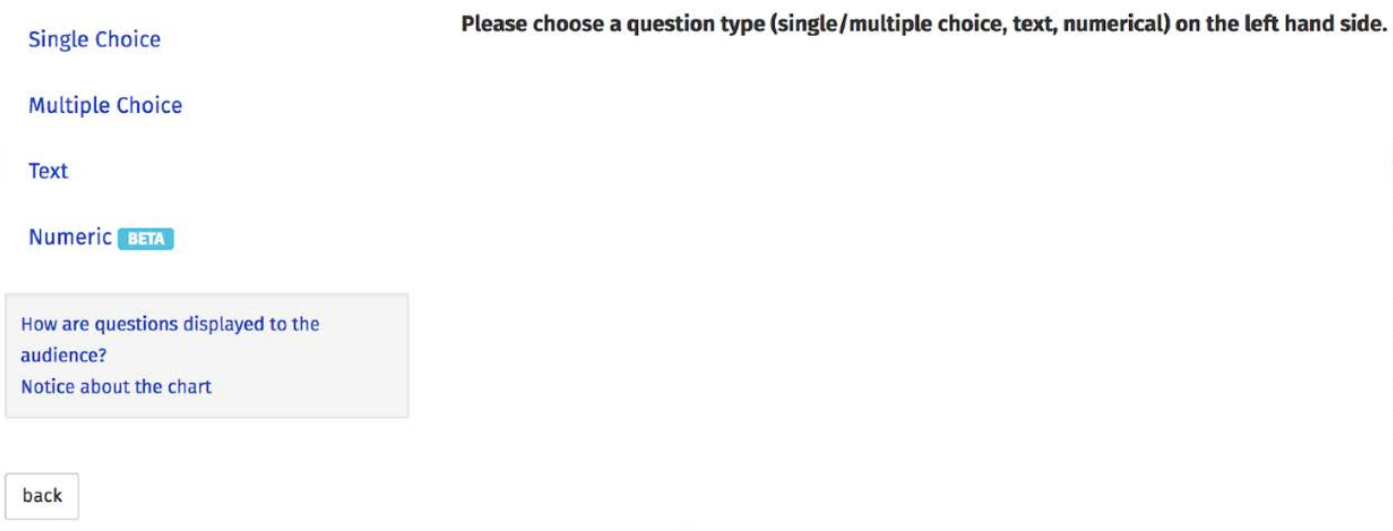

Figure 16. Creating a new question in Pingo.

- There are options to create Single Choice, Multiple Choice, Text and Numeric type questions. As an example, the image below demonstrates the Single Choice option (Figure 2):

\section{Create question}

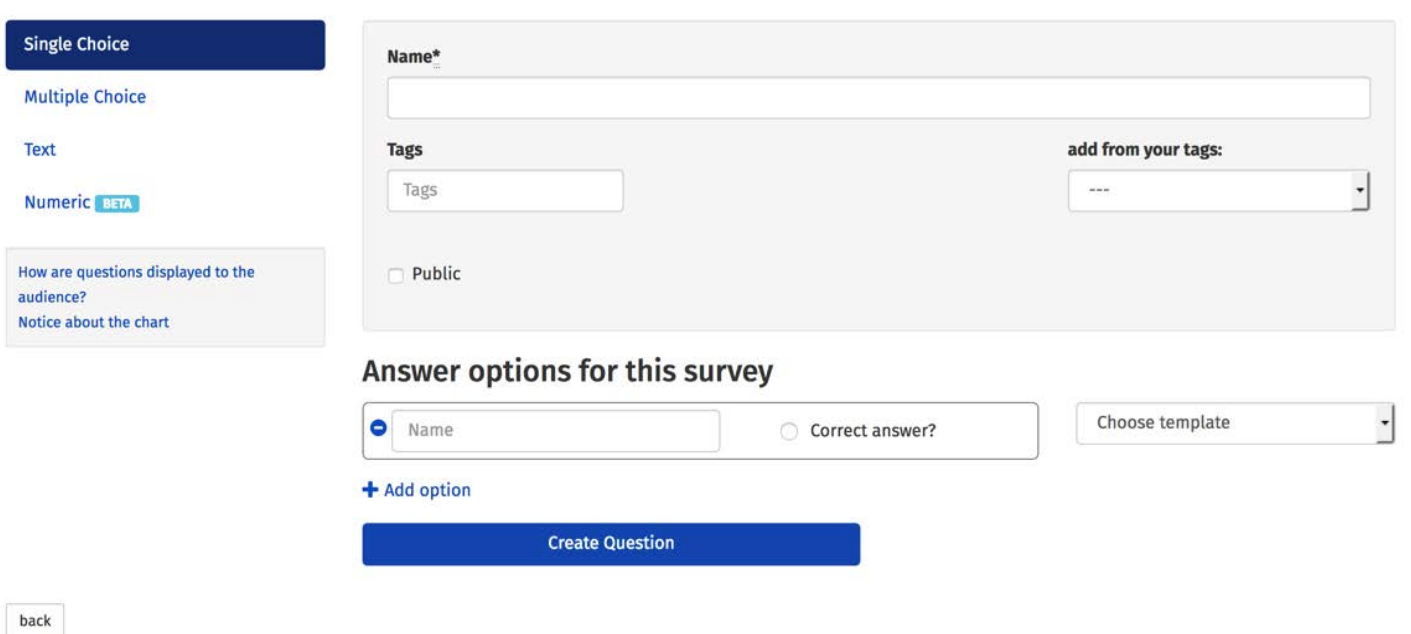

Figure 17. Single Choice question option in Pingo.

- Questions are typed into the Name box above, whereas possible answers are typed into the option boxes below. The correct answer is indicated by ticking the appropriate box. Any required TeX code within questions or answers must be encapsulated within dollar symbols in the usual way. The TeX code is compiled once the question is created. There are also a number of useful templates available from the drop-down menu Choose template. The Tags option is particularly useful if Pingo is used in more than one module/course as it provides a way of separating questions into categories making them easier to manage. The final step is to select Create Question.

- The system is very intuitive which makes creating the other types of questions equally straightforward. Repeating this procedure creates a catalogue of questions saved onto your 
Pingo account. Once a catalogue of questions has been created, a New session must be created to use Pingo in lectures. This is achieved by selecting Survey execution followed by New session. A name must be created for the session and if TeX code is going to be used (including within previously created questions) then the Formula support box must be selected, see Figure 3 below:

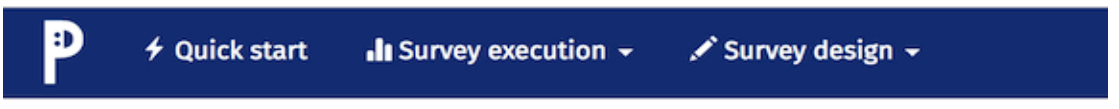

\section{Create new session}

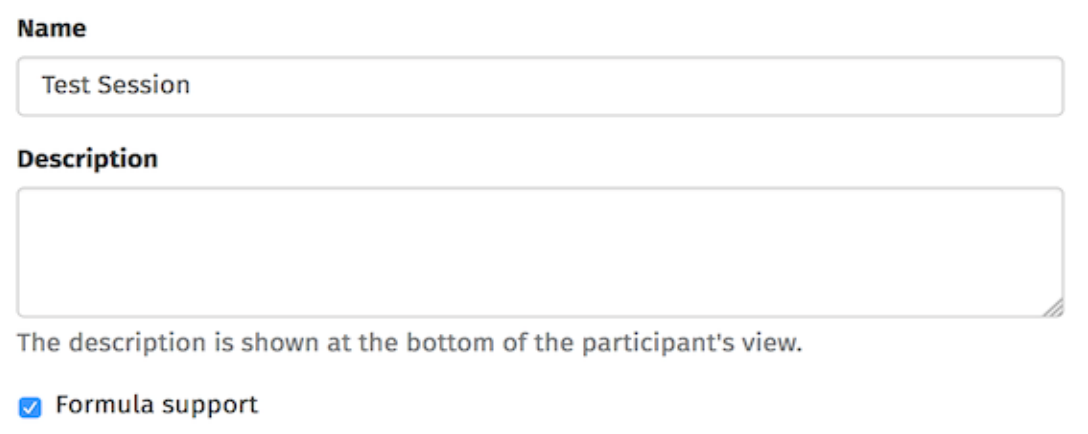

OK

back

Figure 18. Create new session option in Pingo.

- Once a session has been created, it will be assigned a unique number and questions can be set by selecting from your previously created catalogue or by creating questions on-the-spot. These options are accessed on the right-hand side of the following screen (Figure 4):

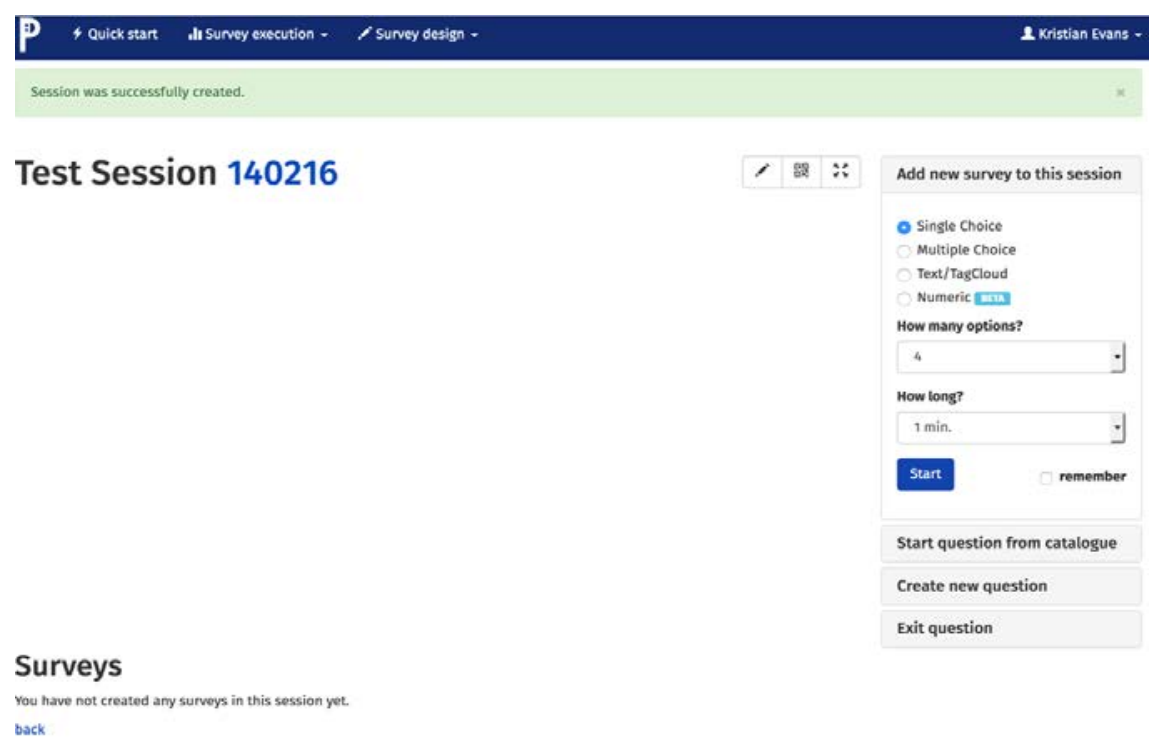

Figure 19. New session page in Pingo.

- Students enter the unique number into their devices to access the session. Once a lecturer sets a question, it will appear both on the screen in the lecture theatre and on the students' devices. There is also a countdown timer to ensure that students know how much time they have. 
- After each question, the results are displayed and lecturers can select the correct answer(s) using the Highlight correct answers option. Figure 5 below shows an example of a Pingo output, along with the highlighted correct answer, for a question asked to a group of third year students studying a complex analysis module:
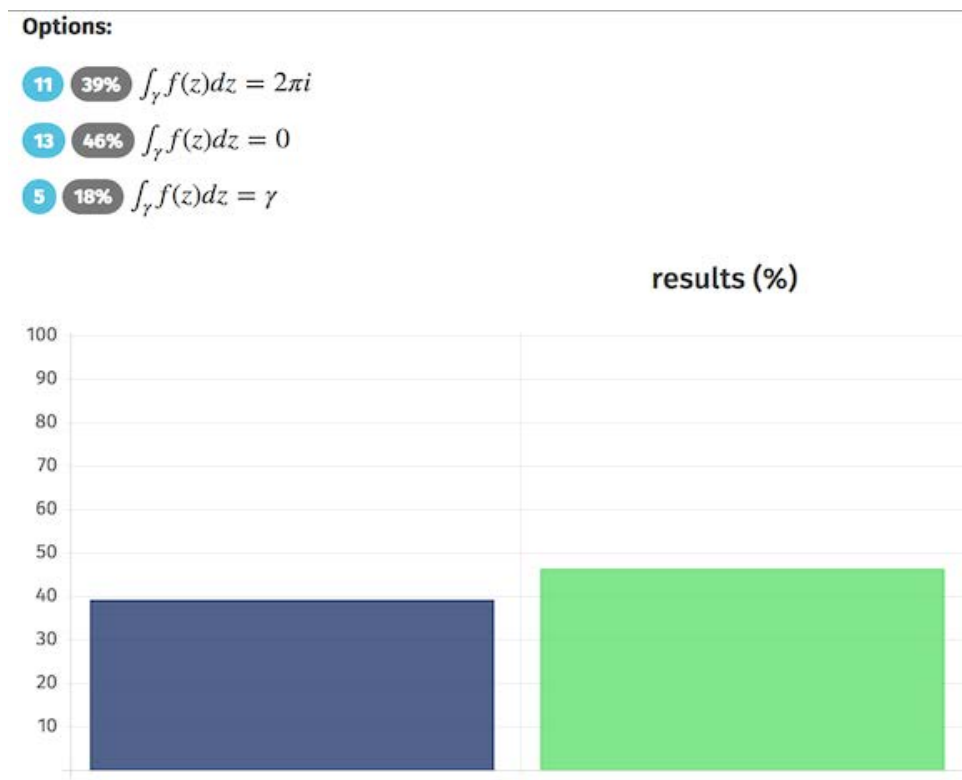

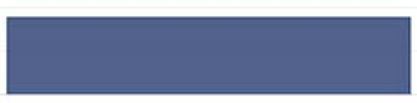

Highlight correct answers

Figure 20. Example question output in Pingo.

- In addition, questions may be imported into Pingo and results of surveys may also be exported. Another useful feature of Pingo is Pingo Remote - this allows the system to be incorporated into presentation software. This option is available from http://trypingo.com

\section{Reflections on Using Pingo}

From a lecturer's perspective, I found Pingo to be extremely useful in obtaining interaction in large group, lecture-style teaching. The results of the surveys also provided instant feedback on what topics were well-understood by the group and which topics required further explanation. This meant that prompt feedback, and further relevant information, could be provided to the students based on this information. I found the system to be user-friendly and it provided an additional tool to track the general progress and understanding of the group, allowing me to adapt my teaching delivery accordingly.

The system has also been very well received by students; to date, I have not received any negative feedback on Pingo. In fact, the questionnaires for the modules in which I have used Pingo have included many positive comments about the system, including:

- "Pingo! I love learning this way, it makes things so much easier for me to understand."

- "Interactive example classes on Pingo are very helpful since you can anonymously answer the questions and consolidate your understanding."

- "Pingo surveys are very useful. I would like all modules to include them."

Furthermore, in the final lecture of my first trial of the use of Pingo I polled the students on their opinions of the system. All responses $(n=20)$ said that they enjoyed the Pingo sessions and all responses $(n=21)$ said that they found the Pingo sessions useful in their learning. Students have 
also commented during staff-student consultative committee meetings how they value the fact that they can anonymously request feedback for specific topics by using Pingo.

I have used Pingo with up to 50 participants without any technical issues. Furthermore, I did not encounter a situation where a student did not have a device compatible with Pingo. If this situation were to arise, pooled university devices could be distributed to such students. In the sessions I used Pingo I never encountered any problems with the WiFi network, but I always checked the network prior to every session.

I have given a variety of presentations with live demonstrations on the use of Pingo, both within Swansea University (2017 SALT Conference) and nationally via the Higher Education Academy (2018 HEA STEM Conference). Following these, some colleagues have adopted the system, again with positive experiences. In particular, one colleague recently remarked that Pingo enables him to "respond to student concerns and questions as soon as possible".

\section{Conclusion}

In my teaching, the use of Pingo has greatly improved the issue of lack of interaction in lectures set out in the Introduction. Prior to using Pingo, I would ask similar questions verbally and rarely receive a response. Using Pingo I have received up to 50 responses. Unfortunately, there are still some students who do not engage and it would be useful and interesting to do study to investigate why this is the case. In my opinion, the main advantages of using Pingo are its TeX-compatibility and the increased interaction obtained, which provides both feedback for students and staff. This feedback can be used by students to improve their learning and by staff to adjust their teaching. As previously mentioned, the system is fee-free and there are no restrictions on participants. The system is simple to set up and use, in particular if catalogues of questions are created before teaching sessions. I think the anonymous nature of answering questions makes it more likely for students to participate, however, the disadvantage of this is providing targeted feedback. A further disadvantage of such systems is that they are clearly reliant on technology working efficiently. As mentioned in the Evaluation, I have not encountered any problems, however, it would be wise to have a nontechnological activity prepared in case of any problems.

\section{Acknowledgements}

I would like to thank the University of Paderborn for creating the Pingo system and permission to write this article. I became aware of the system due to Dr. Mathew Pugh of the Mathematics Department at Cardiff University and I would like to acknowledge this. Finally, I would like to Dr. Gibin Powathil for his input and remarks in using the system.

\section{References}

Bruff, D., 2009. Teaching with Classroom Response Systems: Creating Active Learning Environments. San Francisco: John Wiley \& Sons.

Bates, S., Howie, K. and Murphy, A., 2006. The use of electronic voting systems in large group lectures: challenges and opportunities. The Higher Education Academy, New Directions Issue 2. 10.11120/ndir.2006.00020001

London Mathematical Society, 2011. Use and Misuse of Information and Computer Technology in the Teaching of Mathematics at HE Institutions: Position Statement. Available at: https://www.Ims.ac.uk/sites/Ims.ac.uk/files/Mathematics/policy responses/ICT statement.pdf [Accessed 5 April 2018].

Pingo Sign-up Website, 2018. Available at: http://trypingo.com [Accessed 12 April 2018]. 
Pingo YouTube Channel. 2018. Available at:

https://www.youtube.com/channel/UCoA4vCzvqmc3oRLeEpGfayQ [Accessed 24 July 201].

Pingo Web-based System, 2018. Available at: http://pingo.upb.de [Accessed 12 April 2018].

Shon, H. and Smith, L., 2011. A Review of Poll Everywhere Audience Response System. Journal of Technology in Human Services, 29:3, pp. 236-245. 10.1080/15228835.2011.616475

Steinert. Y. and Snell. L.S, 1999. Interactive lecturing: strategies for increasing participation in large group presentations. Medical Teacher, 21:1, pp. 37-42. 10.1080/01421599980011 